ARTICLE HISTORY: Received: August: 6, 2021 Accepted: September 27, 2021 Published: October 4, 2021

СВОБОДНОЕ КРИВОЛИНЕЙНОЕ ПРОСТРАНСТВО С КРУЧЕНИЕМ.

Шарин Ю.А.

Уральский Государственный Университет

Екатеринбург

\title{
FREE CURVILINEAR SPACE WITH TORSION.
}

Sharin Y.A.

Ural State University

Ekaterinburg

Аннотация. В рамках классической теории поля предложен вариант объединения гравитации и электромагнетизма на основе четырех мерного криволинейного пространства с кручением. Выявлена связь электромагнитного поля с кручением пространства, предложена физическая интерпретация скалярной кривизны пространства как плотности массы материи. Получено решение для собственного состояния криволинейного пространства с кручением, соответствующее электрону. Показана связь уравнений поля с уравнением Шредингера для атома водорода. Найдены космологические решения для расширяющейся Вселенной, проведены оценки средней плотности массы во Вселенной и получены результаты, соответствующие данным астрономических наблюдений.

Abstract. Under the classical field theory, a variant unification of gravity and electromagnetism on the basis of four-dimensional curved space with torsion is proposed. The connection between electromagnetic field and torsion of space is discovered, a physical interpretation of the space scalar curvature as the density of matter mass is proposed. The solution for the eigenstate of a curved space with torsion, corresponding to the electron is obtained. The identification of the field equations as the Schrodinger equation for the hydrogen atom is shown. Cosmological solutions for the expanding Universe are found, the average mass density in the Universe is estimated, and the results corresponding to the data of astronomical observations are obtained.

Ключевые слова: криволинейное пространство, кручение, скалярная кривизна, плотность массы, электрон, Уравнение Шредингера, космологические решения, скрытая масса.

Keywords: curvilinear space, torsion, scalar curvature, density of mass, electron, Schrodinger equation, cosmological solutions, missing mass.

В статье представлено исследование свободных от внешнего воздействия, то есть, собственных состояний криволинейного пространства с кручением определенного вида.

1. Геометрическая интерпретация электромагнитного поля

Предложенный в [1] подход дает возможность в рамках классической теории поля включить гравитацию и электромагнетизм в единую геометрическую схему.

\section{1. Уравнение движения.}

Рассмотрим четырехмерное пространство сигнатуры ( $\square \square \square \square \square()$ ), со связностью

$$
L_{i k l}=I_{i k l}+B_{i k n},(1)
$$

где $T_{i k 1}$ - связность, согласованная с метрикой $g_{i k}$

$$
\Gamma_{i k l}=\frac{1}{2}\left(\frac{\partial g_{i k}}{\partial x^{l}}+\frac{\partial g_{i l}}{\partial x^{k}}-\frac{\partial g_{i k l}}{\partial x^{i}}\right)
$$

а тензор $B_{\text {ikl }}$ имеет вид

$$
B_{i k \mathbb{l}}=\frac{\varepsilon}{\varphi} H_{i k} u_{1}
$$

где $u_{\mathrm{i}}$ - вектор скорости пробной частицы, обладающий свойством $u_{i} u^{\mathrm{i}}=-1, H_{i k^{-}}$обобщенный тензор электромагнитного поля 


$$
\begin{array}{r}
H_{i k}=\frac{1}{\alpha}\left(\frac{\partial \bar{w}_{i}}{\partial x^{k}}-\frac{\partial \bar{w}_{k}}{\partial x^{i}}\right) \\
\bar{w}_{i}=\psi w_{i} w_{i}=u_{i}-\alpha A_{i}, \quad(4)
\end{array}
$$

$A_{\mathbb{1}}$ - вектор-потенциал электромагнитного поля, $\square$ - коэффициент, обеспечивающий правильную физическую размерность, $\psi$ - безразмерный параметр. Отметим, что в силу антисимметричности тензора $B_{i k l}$ по первой паре индексов связность $L_{i \mathrm{kl}}$ также согласована с метрикой. Введенная Картаном [2] антисимметричная по второй паре индексов часть связности, называемая тензором кручения $C_{i k l}$, связана с тензором $B_{i k l}$ соотношениями

$$
\begin{array}{r}
C_{i k l}=\frac{1}{2}\left(B_{i k l}-B_{i l k}\right) \\
B_{i k l}=C_{i k l}+C_{k i i}+C_{i k i}
\end{array}
$$

Уравнение геодезической линии в пространстве со связностью (1)

$$
\frac{\partial u^{\mathbb{Q}}}{\partial s}+L_{k n}^{\mathbb{d}} u^{k} u^{\mathbb{1}}=0
$$

с учетом (3-4) может быть приведено к виду

$$
\frac{\partial u^{\mathbb{Q}}}{\partial s}+F_{\mathbb{1}}^{\mathfrak{i}} u^{k} u^{\mathbb{1}}=\frac{\varepsilon}{\psi-1} F^{i k} u_{k}
$$

где $F_{i k}=\frac{\partial A_{k}}{\partial x^{i}}-\frac{\partial A_{i}}{\partial x^{k}}-$ тензор электромагнитного поля. В случае $\psi=1+\frac{\varpi m c^{2}}{q}$, где $c$ - скорость света, уравнение (7) совпадает с уравнением движения частицы с массой $m$ и электрическим зарядом $q$ в гравитационном и электромагнитном полях.

Таким образом, движение заряженной частицы в гравитационном и электромагнитном полях может быть представлено как движение по геодезической линии в криволинейном пространстве с кручением вида (5).

\section{2. Уравнения поля.}

Прежде чем перейти к уравнениям поля сделаем одно замечание. В уравнении движения $u_{\mathrm{i}}$ - вектор скорости пробной частицы. В уравнениях поля - это некий вспомогательный вектор (обладающий свойством $\left.u_{i} u^{i}=-1\right)$, который нужен только для перехода к величинам, использующимся в теории Максвелла $\left(A_{i}=\frac{1}{\mathbb{a}}\left(u_{i}-\frac{\bar{w}_{i}}{\psi}\right)\right)$, а геометрия пространства полностью определяется метрикой $g_{i k}$ и вектором $\bar{w}_{i}$.

Для построения уравнений, описывающих свободные состояния криволинейного пространства с кручением, выберем действие в виде

$$
\begin{gathered}
D=\iiint \int\left(\mathrm{fw}_{\overline{1}} \bar{w}^{\mathbb{1}} R_{i k} g^{i k}-\frac{\psi^{2}}{4} B_{i k]} B^{i k l}\right) \sqrt{|g|} d x^{0} d x^{1} d x^{2} d x^{a} \\
g=\operatorname{det}\left(g_{i k}\right), \quad(8)
\end{gathered}
$$

где $R_{i k}$ - тензор Риччи риманова пространства

$$
R_{i k}=\frac{\partial \Gamma_{i k}^{n}}{\partial x^{n}}-\frac{\partial \Gamma_{i m}^{n}}{\partial x^{k}}+\Gamma_{i k}^{n} \Gamma_{m m}^{m}-\Gamma_{i m}^{n} \Gamma_{k m}^{m}
$$

$f\left(x^{\mathrm{i}}\right)$ - некоторая безразмерная скалярная функция, физический смысл которой будет ясен ниже. Вариация действия (8) по компонентам вектора $w_{i}$ и тензора $g_{i k}$ приводит к уравнениям

$$
\left\{\begin{array}{l}
R_{i k}-\frac{g_{i k}}{2} R=\frac{4 \pi y}{\mathbb{c}^{4}} T_{i k} \\
H^{i k}{ }_{i k}=\frac{4 \pi}{c} j^{i}
\end{array},\right.
$$




$$
\begin{gathered}
R=R_{i k} g^{i k} \\
T_{i k}=-\frac{c^{4} \alpha^{2}}{8 \pi \gamma f} \frac{1}{\bar{w}_{1} \bar{w}^{l}}\left(H_{i n} H_{k}^{n}-\frac{g_{i k}}{4} H_{n m} H^{n m}\right)-\rho^{[m])} c^{2} \frac{\bar{w}_{i} \bar{w}_{k}}{\bar{w}_{1} \bar{w}^{l}} \\
\rho^{(m)}=\frac{c^{2}}{4 \pi \gamma} R \quad \rho^{[c]} c w^{i} \\
\rho^{(c)}=\frac{f \psi}{2 \pi \varepsilon} R
\end{gathered}
$$

$\square$ - гравитационная постоянная, точка с запятой обозначает риманову производную со связностью (2).

Первое уравнение из (10) представляет собой обобщенное уравнение Эйнштейна для гравитационного поля, второе уравнение является обобщенным уравнением Максвелла, $\square^{(m)}$ играет роль плотности массы, а $\square \square{ }^{(c)}$ играет роль плотности электрического заряда. Закон сохранения энергии-импульса $T_{i k}$ выполняется благодаря тождеству Бьянки, а закон сохранения заряда обеспечивается в силу антисимметричности тензора $H_{i k}$.

Из (11) следует

$$
\frac{p^{([c)}}{p^{f(n x)}}=\frac{2 \gamma \psi}{\alpha c^{2}} f,
$$

то есть функция $f$ - определяет отношение плотности электрического заряда к плотности массы в каждой точке пространства.

Определим массу $m$ и электрический заряд $q$ свободного состояния криволинейного пространства с кручением

$$
\begin{array}{r}
m=\frac{1}{\omega \pi} \int_{0}^{\omega \pi} d x^{0} \rho^{[m i} \sqrt{|g|} d x^{1} d x^{2} d x^{a} \\
q=\frac{1}{\omega \pi} \int_{0}^{\omega \pi} d x^{0} \rho^{[c]} \sqrt{|g|} d x^{1} d x^{2} d x^{3},
\end{array}
$$

где $\square$ - характерное для данного состояния "время жизни", которое должно определяться одновременно с поиском решения уравнений (10).

Из определения плотности массы следует, что физический смысл имеет только пространство с $R \geq 0$, причем при $R \equiv 0$ масса и электрический заряд одновременно обращаются в ноль, это означает то, что существование пространства с нулевой массой и отличным от нуля электрическим зарядом невозможно. В то же время, при $R>0$ масса будет отлична от нуля, но в случае, если функция $f$ является знакопеременной, суммарный электрический заряд может быть равен нулю и электрически нейтральное состояние пространства с ненулевой массой возможно.

Определение вида этой функции выходит за рамки предложенной схемы, что делает данное рассмотрение неполным. Будут представлены только некоторые частные случаи вида функция $f$.

Полагая $\psi=$ const уравнения (10) можно переписать в виде

$$
\left\{\begin{array}{l}
R_{i k}-\frac{g_{i k}}{2} R=-\frac{1}{2 f w_{l} w^{l}}\left(h_{i n} h_{k}{ }^{n}-\frac{g_{i k}}{4} h_{n m} h^{n m}\right)-\frac{w_{i} w_{l k}}{w_{l} w^{l}} R \\
h^{i k}{ }_{i k}=2 f R w^{i}
\end{array},\right.
$$

где $h_{i k}=\frac{\partial w_{l}}{\partial x^{k}}-\frac{\partial w_{k}}{\partial x^{i}}$.

\section{2. Стационарное свободное пространство}

Рассмотрим свободное состояние криволинейного пространства с кручением, полагая метрику $g_{i k}$ и вектор $w_{i}$ не зависящими от времени, тогда $\frac{1}{\sigma \tau} \int_{0}^{c \tau} d x^{0} \equiv 1$, а "время жизни" такого состояния можно считать равным бесконечности.

\section{1. Электрон.}

В [1] рассмотрены уравнения (14) для случая $f \equiv 1$. Найдено сферически симметричное решение для гравитационного и электрического полей 


$$
\begin{aligned}
& \nu=\chi=-\lambda=-\mu \\
& e^{v}=\frac{r}{r+r_{0}}
\end{aligned}
$$

где введены следующие обозначения

$$
\begin{gathered}
g_{00}=-e^{v} \quad g_{11}=e^{\lambda} \quad g_{22}=r^{2} e^{\mu} \quad g_{33}=r^{2} e^{\mu} \sin ^{2} \theta \\
w_{0}=e^{X}(16)
\end{gathered}
$$

$r_{0}$ - константа интегрирования. Подставляя решение (15) в (13), получаем

$$
r_{0}=\frac{2 \gamma m}{c^{2}} \quad \alpha=\frac{\varphi r_{0}}{q}=\frac{2 \gamma m \varphi}{c^{2} q} .
$$

Тогда, в случае $\psi=1$ выражения для гравитационного и электрического потенциалов

$$
\begin{gathered}
G=-\frac{c^{2}}{2}\left(1+g_{00}\right)=-\frac{\gamma m}{\gamma+\gamma_{0}} \\
A_{0}=\frac{1}{\alpha}\left(1-w_{0}\right)=\frac{q}{\gamma+r_{0}}
\end{gathered}
$$

соответствуют закону всемирного тяготения Ньютона и закону Кулона. Отклонения от этих законов становятся заметны только вблизи центра симметрии на расстояниях порядка $r_{0}$. Для электрона, например, $r_{0} \sim 10^{-55} \mathrm{~cm}$, что означает практическую невозможность экспериментальной проверки таких отклонений.

Также в [1] проведена оценка асимптотики аксиально-симметричного решения уравнений (14) для магнитного поля и получено гиромагнитное отношение

$$
\frac{S}{M}=\frac{m e}{q}
$$

где $M$ - магнитный момент, $S$ - момент импульса.

Поскольку, гиромагнитное отношение (19) характерно только для электрона, то электрон может рассматриваться как стационарное, аксиально-симметричное свободное состояние криволинейного пространства с кручением при условии подобия распределения плотностей электрического заряда и массы $(f \equiv 1)$.

2.2. Атом водорода.

Пренебрегая гравитационным полем, рассмотрим второе уравнение из (10), считая, вектор $w_{i}$ не зависящим от координат, метрику евклидовой, а параметр $\psi$ зависящим от координат.

Выберем функцию $f$ в виде

$$
f=\frac{1}{2 R}\left(a_{1}+\frac{a_{2}}{r}\right),(20)
$$

где $a_{1}$ и $a_{2}$ - некоторые не зависящие от координат параметры, при этом первый член в скобках соответствует распределению электрического заряда электрона, второй - протона.

Тогда второе уравнение из (10) принимает вид

$$
\Delta \psi+\left(a_{1}+\frac{a_{2}}{T}\right) \psi=0,
$$

где $\Delta$ - оператор Лапласа. 
Если $a_{1}=\frac{2 m E}{\hbar^{2}}, a_{2}=\frac{2 m e^{2}}{\hbar^{2}}$, где $h=\frac{\mu}{2 \pi}, h$ - постоянная Планка, $m$ - масса $e$ - заряд электрона, $E-$ полная энергия электрона, то уравнение (21) совпадает с Уравнением Шредингера для атома водорода, в котором ж является волновой функцией электрона.

Из определения (13) с учетом (20) и определения плотности электрического заряда (11) получаем полный заряд атома водорода

$$
q=\frac{m}{2 \pi h^{2}} \int_{0}^{\infty}\left(E+\frac{e^{2}}{r}\right) \Phi(r) d r,
$$

где $\Phi(r)=r^{2} \int_{0}^{\pi} \quad \int_{0}^{2 \pi} \psi \sin ^{2} \vartheta \quad d \vartheta d \phi$. Используя известную теорему

$$
\int_{0}^{\infty} \frac{1}{r} \Phi(r) d r=\frac{1}{r_{1}} \int_{0}^{\infty} \Phi(r) d r
$$

где $0<r_{1}<\infty_{и з}(27)$ получаем

$$
q=\frac{m}{2 \pi h^{2}}\left(E+\frac{\theta^{2}}{r_{1}}\right) \int_{0}^{\infty} \Phi(r) d r
$$

и в случае $E=-\frac{e^{2}}{\widetilde{x}_{1}}$ полный электрический заряд атома водорода будет равен нулю. Это означает, что для любого значения полной энергии электрона $E$ всегда найдется такое $r_{1}$, при котором обеспечивается электрическая нейтральность атома водорода.

Таким образом, атом водорода можно рассматривать как собственное состояние криволинейного пространства с кручением при выполнении условия (20).

3. Космология свободного криволинейного пространства

Рассмотрим свободное однородное изотропное состояние криволинейного пространства, пренебрегая электромагнитным полем, то есть, полагая, что $A_{i}$ uf $\mathrm{c}$ одинаковой скоростью стремятся к нулю во всем пространстве. Тогда второе уравнение из (14) становится тривиальным, а первое принимает рассмотренный в [34] вид

$$
R_{\text {ik }}-\frac{g_{\text {ik }}}{2} R=u_{i} u_{k} R \text {. }
$$

\section{1. Закрытая модель}

Воспользуемся стандартной заменой переменных (полагая остальные компоненты равными нулю)

$$
\begin{array}{cccc}
c d t=a d \eta & a=a(\eta) \quad r=a \sin \gamma \quad(0 \leq \eta \leq 2 \pi & 0 \leq X \leq \pi) \\
\theta_{00}=-a^{2} \quad g_{11}=a^{2} & g_{22}=a^{2} \sin ^{2} \chi \quad g_{33}=a^{2} \sin ^{2} \chi \sin ^{2} \vartheta & (0 \leq \vartheta \leq \pi) \\
u_{0}=a,(26) &
\end{array}
$$

выражения для компоненты $u_{0}$ следует из условия $u_{i} u^{i}=-1$.

Тогда уравнение (30) принимает вид

$$
2 a^{m} a-a^{12}+a^{2}=0,
$$

где штрих обозначает производную по $\square$. Уравнение (22) имеет решение

$$
a=a_{0}(1-\cos \eta)
$$

где $a$ - радиус кривизны пространства, $a_{0}$ - константа интегрирования. Решение (28) совпадает с решением для закрытой космологической модели в ОТО, однако, получено оно без каких бы то ни было дополнительных предположений относительно вида тензора плотности энергии-импульса. 


\section{2. Открытая модель}

В этом случае

$$
\begin{gathered}
c d t=a d \eta \quad a=a(\eta) \quad r=a \operatorname{sh} \chi \quad(0 \leq \eta<\infty \quad 0 \leq X<\infty) \\
g_{00}=-a^{2} \quad g_{11}=a^{2} \quad \begin{array}{c}
g_{22}=a^{2} \operatorname{sh}^{2} \chi \quad g_{a 3}=a^{2} \operatorname{sh}^{2} \chi \sin ^{2} \vartheta \\
u_{0}=a,(29)
\end{array}
\end{gathered}
$$

а уравнение (29) принимает вид

$$
2 a^{m} a-a^{\prime 2}-a^{2}=0
$$

и имеет решение

$$
a=a_{0}(\operatorname{ch} \eta-1) .
$$

Таким образом, уравнение (25) включает в себя решение, как для закрытой, так и для открытой космологической модели. При этом понятие критической плотности массы отсутствует.

В качестве критерия отбора модели, которая соответствует действительности, можно взять величину $t H$, где $t=\frac{1}{e} \int a d \eta$ - время, прошедшее с начала расширения Вселенной, $H=c \frac{\mathscr{a}^{\alpha}}{a^{2}}$ - параметр Хаббла.

Для закрытой модели

$$
t H=(\eta-\sin \eta) \frac{\sin \eta}{\left(1-\cos \eta \eta^{2}\right.}
$$

для открытой модели

$$
t H=(\operatorname{sh} \eta-\eta) \frac{\operatorname{sh} \eta}{\operatorname{sch} \eta-1)^{2}} .
$$

Поскольку

$$
\begin{array}{r}
(\eta-\sin \eta) \frac{\sin \eta}{(1-\cos \eta)^{2}} \leq \frac{2}{a} \\
(\operatorname{sh} \eta-\eta) \frac{\operatorname{sh} \eta}{(\operatorname{col} \eta-1)^{2}} \geq \frac{2}{a^{\prime}} \quad \text { (34) }
\end{array}
$$

то в случае $t H<\frac{2}{g}$ - реальности соответствует закрытая, в случае $t H>\frac{2}{a}$ - открытая модель Вселенной.

Используя оценку «возраста» Вселенной $t \sim 5 \cdot 10^{17}$ сек, сделанную астрономами на основе времени, необходимого для формирования шаровых звездных скоплений, и «возраста» самых старых звезд и оценку параметра Хаббла $H \sim 2 \cdot 10^{-18}$ сек $^{-1}$ получаем $t H \sim 1$, что соответствует открытой модели Вселенной.

\section{3. Скрытая масса}

Возникновение проблемы скрытой массы связано с обнаружением Ф. Цвикки избыточных скоростей галактик из скопления в созвездии Волосы Вероники. Впоследствии исследование орбитальных скоростей звезд плоской составляющей спиральных галактик, и так называемого «рентгеновского газа» в скоплениях галактик подтвердило наличие во Вселенной темной материи, не излучающей электромагнитных волн и не участвующей в формировании звезд, но обладающей массой, благодаря которой она и проявляет себя.

По оценкам астрономов [5] суммарная плотность массы во Вселенной с учетом скрытой массы составляет $10^{-30} \frac{\vec{z}}{\mathrm{Ca}^{3}}<\rho<10^{-29} \frac{z}{\mathrm{Cat}^{3}}$.

В рамках развиваемой теории любое пространство положительной кривизны должно обладать массой, поэтому в качестве скрытой массы мы можем рассматривать массу искривленного пространства Вселенной. 
Скрытой эта масса является в силу того, что, рассмотренные в предыдущем разделе формы пространства не связаны с электромагнитным полем, то есть, «не видимы».

В соответствие с определениями (11) мы имеем с учетом решения (28) для закрытой модели плотность массы искривленного пространства

$$
\rho=\frac{3 c^{2}}{2 \pi \gamma a_{0}^{2}}(1-\cos \eta)^{-3}
$$

Используя время, прошедшее с начала расширения Вселенной $t=\frac{\varpi_{0}}{c}(\eta-\sin \eta)$, мы можем переписать выражение (35) в виде

$$
\rho=\frac{a}{2 \pi \gamma t^{2}} \frac{(\eta-\sin \eta)^{2}}{(1-\cos \eta)^{2}}
$$

На этапе расширения Вселенной $(0 \leq \eta \leq \pi)$ мы имеем $0.2<\frac{(\eta-\sin \eta)^{2}}{(1-\cos \eta)^{3}}<1.24$, тогда, учитывая оценку «возраста» Вселенной $t \sim 5 \cdot 10^{17}$ сек получаем оценку плотности массы искривленного пространства $p \sim 10^{-29} \frac{2}{e^{3}}$.

В открытой модели с учетом решения (31) скалярная кривизна

$$
R=\frac{6}{a^{3}}\left(a^{m}-a\right)=\frac{6}{a_{0}^{2}(c h n-1)^{3}}
$$

также положительна и мы можем определить плотность массы искривленного пространства

$$
\rho=\frac{3 c^{2}}{2 \pi \gamma a_{0}^{2}}(\operatorname{ch} \eta-1)^{-3}
$$

Используя параметр Хаббла $H=\frac{c}{a_{0}} \frac{\operatorname{sh} \eta}{(\operatorname{ch} \eta-1)^{2}}$, мы можем переписать (38) в виде

$$
\rho=\frac{3 H^{2}}{2 \pi \eta} \frac{\operatorname{ch} \eta-1}{\operatorname{sh}^{2} \eta}
$$

Поскольку $0<\frac{\operatorname{ch~} \eta-1}{\operatorname{sh}^{2} \eta} \leq 0,5$, причем ноль достигается только при $\eta \rightarrow \infty$, для грубой оценки плотности массы мы можем считать $\frac{\operatorname{ch} \eta-1}{\operatorname{sh}^{2} \eta} \sim 0,25$. Тогда, учитывая оценку параметра Хаббла $H \sim 2 \cdot 10^{-18}$ сек ${ }^{-1}$, получаем $\rho \sim 10^{-29} \frac{z}{e^{3}}$.

Таким образом, грубые оценки плотности массы криволинейного пространства в обеих моделях совпадают и соответствуют астрономическим оценкам средней плотности массы во Вселенной с учетом скрытой массы.

\section{Заключение}

Как уже отмечалось выше, предложенный вариант теории криволинейного пространства с кручением является неполным из-за неопределенности вида функции $f$. Это связано с тем, что данная функция определяет состав и виды материи, которые содержит в себе пространство. В статье рассмотрены варианты:

- пространство содержит единственный электрон $f \equiv 1$;

-пространство содержит единственный атом водорода $f=\frac{1}{2 R} \frac{2 m}{\pi^{2}}\left(E+\frac{e^{2}}{g}\right)$;

- пространство содержит только темную материю $f \rightarrow 0$.

Очевидно, что определение состава материи, содержащейся в пространстве, а, следовательно, вида функции $f$, выходит за рамки теории поля.

\section{Список литературы:}

1. Шарин Ю.А. Электрон - как собственное состояние криволинейного пространства с кручением//Журнал «Известия вузов. Физика.» - 1998,- №5, - с. 56-60.

2. Cartan E.// Compt. Rend. Acad. Sci. (Paris). - 1922, Vol. 174, p. 437-593.

3. Шарин Ю.А. Космология криволинейного пространства с кручением// Журнал «Известия вузов. 
Физика.» - 1999,- №11, - с. 72-74.

4. Шарин Ю.А. Скрытая масса во Вселенной и кривизна пространства// Журнал «Известия вузов. Физика.» - 2002,- №11, - с. 85-87.

5. Новиков И.Д. Эволюция Вселенной. - М.: Наука, 1990, - с. 52.

References:

1. Sharin Y.A. Electron-as an eigenstate of a curved space with torsion//Russian Physics Journal. - 1998,- №5, - p. 56-60.

2. Cartan E.// Compt. Rend. Acad. Sci. (Paris). - 1922, Vol. 174, p. 437-593.

3. Sharin Y.A. Cosmology of curved space with torsion//Russian Physics Journal. - 1999,- №11, - p. 72-74.

4. Sharin Y.A. Hidden mass in the Universe and the curvature of space //Russian Physics Journal. - 2002,- №11, C. 85-87.

Novikov I.D. Evolution of the Universe -M. Science, 1990,- p. 52. 